\title{
A HYBRID HEURISTIC ALGORITHM FOR SCHOOL DISTRICT DIVISION
}

\author{
Xue $\mathrm{He}^{1,2}$, Bo Wei ${ }^{1,3, *}$ \\ ${ }^{1}$ Guangxi Key Laboratory of Spatial Information and Geomatics, Guilin University of Technology, Jiangan Road, Guilin, China - \\ (hexuesnowy, superweibo)@glut.edu.cn \\ ${ }^{2}$ Nanning Land Surveying and Mapping Geographic Information Center, Jinchun Road, Nanning, China \\ ${ }^{3}$ College of Geomatics and Geoinformation, Guilin University of Technology, Jiangan Road, Guilin, China
}

\section{Commission VI, WG VI/4}

KEY WORDS: Single-School District Division, Multi-School District Division, Hybrid Heuristic, Multi-Start Iterative Local Search, Simulated Annealing, Neighborhood Search, Spatial Continuity

\begin{abstract}
:
Single-school and multi-school district divisions are the two main ways to balance educational resources for enrollment in primary and secondary schools. A hybrid heuristic algorithm (M-ILS-SA) for school district division is proposed based on the combination of a Multi-Start Iterative Local Search (M-ILS) algorithm and a Simulated Annealing (SA) algorithm. According to the principle of "school grouping first and student assigning second", a K-Medoids model is first used to implement school grouping. Then, the initial solution for each run of ILS that starts is generated by the region growth algorithm. After completing the neighborhood search, the SA algorithm is finally used to choose the optimal solution from the historically generated school districts identified by ILS. The experimental results show that the proposed M-ILS-SA algorithm can effectively reduce the total elapsed time and the number of over-enrolled students in each school district, and ensure spatial continuity in both single-school and multi-school district divisions.
\end{abstract}

\section{INTRODUCTION}

Single-school district division and multi-school district division are the two main methods of controlling enrollment in primary and secondary schools. Single-school district division is a special case of the multi-school district division problem, in which the number of school districts is equal to the number of schools. The problem of school district division is closely related to the spatial layout of schools, the allocation of educational resources, and transportation.

A linear programming model is usually used to solve the problem of school district division. A general mathematical model in the school district was established by (Koenigsberg, 1968) to examine student integration policies. An experimental study using linear programming was proposed by (Mckeown, Workman, 1976) to design attendance. Based on the prediction of the urban census, a linear programming model was used by (Jennergren, Obel, 1980) to divide school districts. Some researchers have also adopted a linear programming model to avoid overcrowding in the district (Franklin, Koenigsberg, 1973, Schoepflea, Churchb, 1991, Taylor et al., 1999). However, the linear programming model is very complicated in terms of computing, and its results of school district division cannot satisfy the requirement of spatial continuity.

Since the problem of school district divisions is a typical zoning problem, a clustering tree was established by (Guo, 2008, Guo, Jin, 2011) based on the related improved algorithm of agglomerative hierarchical clustering analysis. By extending Automatic Zoning Procedure (AZP), the search process was prevented from falling into a local optimum (Openshaw, 1977, Kim et al., 2016). A service area division problem similar to the school district problem was described by (Ko et al., 2015), which they designed a neighborhood search operator to iteratively improve the service partition in the simulated annealing algorithm framework. However, if only one neighborhood search operator is used, it is easy for the search process to fall into a local optimum.

At present, the Simulated Annealing (SA) algorithm has been used for partition optimization and has shown good global optimization performance (Ricca, Simeonea, 2008, RincónGarcía et al., 2013, Rincón-García et al., 2017, Assad, Deep, 2018). In this contribution, we design a hybrid heuristic algorithm (M-ILS-SA) based on SA, which mainly includes school grouping, initial solution construction, neighborhood search operator optimization, and SA algorithm modeling and solving. The designed algorithm is applied to the problems of single-school and multi-school district divisions, and shows it can solve the main defect of the existing algorithms for school district division that the partitions cannot guarantee spatial continuity and it is easy to fall into a local optimum.

\section{DESCRIPTION OF THE SCHOOL DISTRICT DIVISION PROBLEM}

The school district division problem is described as follows: Divide set $N$ into $k$ areas, which are denoted by the set $R=\left\{r_{l}\right.$, $\left.r_{2}, \ldots, r_{k}\right\}$, and achieve the shortest student enrollment distance based on the following specific constraints (Kong et al., 2017):

$$
\begin{gathered}
N=r_{1} \cup r_{2} \cup \cdots \cup r_{k} \\
r_{x} \cap r_{y}=\varnothing, \forall r_{x}, r_{y} \in R, r_{x} \neq r_{y}
\end{gathered}
$$

* Corresponding author 


$$
\begin{gathered}
r_{x} \neq \varnothing, \forall r_{x} \in R \\
\sum_{x \in r_{y}} p_{x} \leq \sum_{x \in r_{y}} q_{x}, \forall r_{y} \in R \\
A \leq \sum_{x \in r_{y}} p_{x} \leq B, \forall r_{y} \in R \\
r_{x} \text { spatial continuity, } \forall r_{x} \in R
\end{gathered}
$$

where $\quad N=$ all spatial units

$x, y=$ spatial unit

$p_{x}=$ the number of student enrollment within $x$

$q_{x}=$ the number of school enrollment within $x$

$A, B=$ two constants

In this paper, we use the linear distance between two points as the basis of distance calculation. According to the probability assigned to the high-quality school, the distance from the student to the nearest school and the high-quality school can be weighted to calculate the enrollment distance. That is, the total student enrollment distance of a unit $i$ in a district $k$ can be defined as $D_{i k}$ :

$$
D_{i k}=p_{i} \times\left[\sum_{j \in H_{k}}\left(d_{i j} \times \frac{q_{j}}{\sum_{j \in H_{k}} q_{j}}\right)+\min \left\{d_{i j}, \forall j \in S_{k}\right\} \times\left(1-\sum_{j \in H_{k}} \frac{q_{j}}{\sum_{j \in H_{k}} q_{j}}\right)\right], i \in N_{k}
$$

where $\quad i=$ a spatial unit

$p_{i}=$ the number of student enrolment in $i$

$j=$ a school

$q_{j}=$ the number of school enrollment within $j$

$d_{i j}=$ distance from $i$ to $j$

$k=$ a district

$H_{k}=$ the set of high-quality schools in $k$

$S_{k}=$ the set of schools in $k$

$N_{k}=$ the set of spatial units in $k$

\section{HYBRID HEURISTIC ALGORITHM}

In this paper, we propose a hybrid heuristic (M-ILS-SA) algorithm to solve the problem of school district division. The proposed M-ILS-SA algorithm is a combination of the MultiStart Iterative Local Search (M-ILS) algorithm and the SA algorithm. The principle of the M-ILS-SA algorithm is to construct multiple different initial solutions, to record all the discovered partitions after a neighborhood search operation, to implement globally optimize, and to solve the partition scheme by constructing a SA model.

\subsection{School Grouping Model}

The number of schools is a fixed value. In this paper, according to the principle of the K-Medoids model (Lai, Fu, 2011, Sheng, 2006), we construct a K-Medoids model based on the schools' point layer of ArcGIS, in which the schools are grouped under certain constraint conditions with spatial aggregation as objective (Kong et al., 2017).

In the K-Medoids model, $K$ center points are randomly generated first and grouping results are obtained according to the K-Medoids algorithm. Then, we determine whether the grouping results satisfy the constraint conditions, and find $m$ grouping results that satisfy the constraint conditions. Finally, the final grouping results are obtained by the shortest total distance from each school to the central school in the group as the objective condition.

\subsection{Constructing Initial Solution}

Based on the results of the school grouping, the seed region growing algorithm is used to construct an initial solution. The steps are as follows:

1. Set the partition number of the spatial unit, where a school is located as the school group number and as an initial seed point.

2. Construct adjacent unpartitioned units according to the current seed point. Get the list of the unpartitioned units adjacent to each partition.

3. Randomly select a unit from the list of unpartitioned units, and assess whether the unit violates the partition restriction conditions. If it does not violate this condition, the unit will be added to the objective partition and the current partition updated. Otherwise, the unit will not be added.

4. Repeat step 3 until we go through the whole list of unpartitioned units adjacent to each partition obtained by step 2.

5. After completing step 4, use the boundary unit of a current partition as a new seed point to continue steps $2-4$ until all unpartitioned units that can be added are added into their corresponding objective partitions.

6. Make the following judgments for unpartitioned units that do not meet the conditions: (1) If the partition numbers of all adjacent units of a unpartitioned unit are the same, the unpartitioned units will be added into that numbering objective partitions. (2) If there are different partition numbers for the adjacent units of an unpartitioned unit, assume that the unpartitioned units will be added into different partitions, and determine whether the number of school enrollment is greater than or equal to the number of student enrollment. If all the conditions are satisfied or all the conditions are not satisfied, the unpartitioned units will be randomly added into their adjacent partitions. If only partial conditions are satisfied, the unpartitioned units will be randomly added into the partitions that satisfy the conditions.

Since each step has only one unit that satisfies the conditions and be added into one partition when constructing an initial solution by the existing M-ILS-SPP algorithm (Kong et al., 2017), we attempt to add all the adjacent unpartitioned units that satisfy the conditions into their corresponding objective partitions in step 3. The purpose of this step is to improve the efficiency of constructing an initial solution and save running time. Additionally, a random selection method used in this step is to obtain different initial partition schemes under multiple repetitions, and thus different initial partition schemes can be provided for the M-ILS algorithm.

The initial partition scheme obtained by the above steps has spatial continuity, but some partitions do not satisfy the constraints. This problem can be solved by using the following neighborhood search operators.

\subsection{Neighborhood Search Operator}

Neighborhood search operators are used to adjust the partitions if the initial partition scheme does not satisfy the constraint 
conditions. The following four neighborhood search operators presented in the M-ILS-SPP algorithm are used:

1. 1-0 move: moving a boundary unit of the partition to be improved to the adjacent partition, involving the changes of two partitions.

2. 1-1 move: the partition to be improved moves into a neighboring unit while removing a boundary unit, involving the changes of two or three partitions.

3. 2-1 move: the partition to be improved moves into one neighboring unit, while removing two boundary units, involving the changes of two or three partitions.

4. 1-1-1 move: moving three boundary units at the same time, involving multiple partitions.

Especially for single-school district division, based on a 1-0 move, the following two improved neighborhood search operators for four partitions and eight partitions, (1-0) ${ }^{4}$ move and $(1-0)^{8}$ move, respectively, are designed to expand the search scope and improve the search speed:

1. (1-0 $)^{4}$ move: randomly select four partitions to be improved, and select one boundary unit from them, respectively, and move the selected boundary unit into an adjacent partition.

2. $(1-0)^{8}$ move: randomly select eight partitions to be improved, select one boundary unit from them, respectively, and move the selected boundary unit into an adjacent partition.

Whether multi-school district division or single-school district division, random selection of neighborhood search operator is adopted in the neighborhood search process and only one search operator is chosen randomly in each search process. For multi-school district division, the search operator is randomly chosen from 1-0 move, 1-1 move, 2-1 move, and 1-1-1 move. For single-school district division, using the 2-1 move is not appropriate because it is difficult to find two units that can move simultaneously in a partition due to a large number of school districts and a small number of units. Therefore, the search operator is randomly chosen from 1-0 move, 1-1 move, 1-1-1 move, (1-0) move, and (1-0) ${ }^{8}$ move in single-school district division.

In the neighborhood search procedure, an objective value evaluation function to determine whether to accept the neighborhood solution is used as follows (Kong et al., 2017):

$$
F\left(S, S^{\prime}\right)=D_{S}-D_{S^{\prime}}+\alpha\left(O_{S}-O_{S^{\prime}}\right)
$$

where $\quad S=$ current solution

$S^{\prime}=$ neighborhood search solution

$D_{S}=$ current total student enrollment distance

$D_{S^{\prime}}=$ total student enrollment distance after

neighborhood search

$\alpha=$ a coefficient

students

$O_{S}=$ the total number of current over-enrolled

$O_{S^{\prime}}=$ the total number of over-enrolled students after neighborhood search

When $F\left(S, S^{\prime}\right)>0$, the neighborhood solution is accepted. The purpose of using this evaluation function is to reduce the student enrollment distance and the number of over-enrolled students at a cost of a smaller increase of student enrollment distance.

\subsection{SA Algorithm}

The SA algorithm proposed by (Kirkpatrick et al., 1983) has become one of the most popular heuristic methods to solve the combinatorial optimization problem. The SA algorithm simulates the physical annealing process, which starts from a given initial high temperature, a Metropolis sampling strategy with probabilistic jump characteristics is used to randomly search in the solution space, and a global optimal solution is obtained by a repeated sampling process with decreasing temperature. The advantage of the SA algorithm is to avoid falling into a local optimum (Liu et al., 2015). In this paper, we use the number of current student enrollment in each partition as the initial temperature $T_{0}$, and use the number of school enrollment in each partition as the termination temperature $T_{f}$. The optimization problem is described as follows:

$$
F(i)=D_{i}+\alpha O_{i}
$$

where $\quad i=$ current status

$D_{i}=$ total student enrollment distance from each unit in a partition to the nearest school

$$
O_{i}=\text { the number of over-enrolled students }
$$$$
\alpha=\text { a coefficient }
$$

After the SA optimization is completed, an optimal objective function is introduced in the SA model to obtain the optimal partition results. The optimal objective function is as follows (Kong et al., 2017):

$$
\begin{gathered}
F=\operatorname{Minimize}(M \times C+D) \\
D=\sum_{k \in K} \sum_{i \in N_{k}} D_{i k}
\end{gathered}
$$

where $\quad M=$ a sufficiently large integer

$C=$ the number of over-enrollment in the partition

scheme

$$
\begin{aligned}
& D=\text { the total student enrollment distance } \\
& i=\text { a spatial unit } \\
& k=\text { a district } \\
& D_{i k}=\text { the total student enrollment distance of } i \text { in } k
\end{aligned}
$$

\subsection{Procedure of School District Division}

Multi-school districts and single-school districts are divided according to the principle of "school grouping first and student assigning second". The basic procedures of the two school district division are the same: the K-Medoids model is used first to group the schools, and then the proposed M-ILS-SA algorithm is used for partitioning. The main procedures for multi-school district division are as follows:

1. School grouping. The K-Medoids model is constructed for grouping schools based on the schools' point layer of ArcGIS. The grouping process ensures that each school point has spatial clustering. The difference between the number of school enrollment and the average number of school enrollment in each group cannot exceed the specified limit, and there is at least one high-quality school in each group. 
2. Construction of initial solution. Use the spatial unit where the school is located as the initial seed area, and construct the initial solution by using the seed region growing algorithm. The obtained initial solution may not satisfy the constraint conditions, but at least guarantee the spatial continuity of the partitions. This process is carried out in the framework of the M-ILS algorithm framework and can get $M$ initial partition schemes.

3. Design of neighborhood search operators. Several neighbor search operators, such as 1-0 move, 1-1 move, 21 move, and 1-1-1 move, are designed to adjust each initial partition if it does not satisfy the constraint conditions.

4. Optimization of partition. Use the SA model to further optimize the partition results of the neighborhood search, and to find more partition schemes that satisfy the constraints from the global perspective, so as to determine the optimal partition scheme.

Compared with the procedures of multi-school district division, there are two differences in the procedures of single-school district division. One is in the school grouping step: there is no need to group schools and each school is a group. The other is in the step of design of neighborhood search operators, which the neighborhood search operators are replaced by 1-0 move, 1 1 move, 2-1 move, 1-1-1 move, $(1-0)^{4}$ move and $(1-0)^{8}$ move. The basic flowchart of single-school and multi-school district divisions is shown in Figure 1.

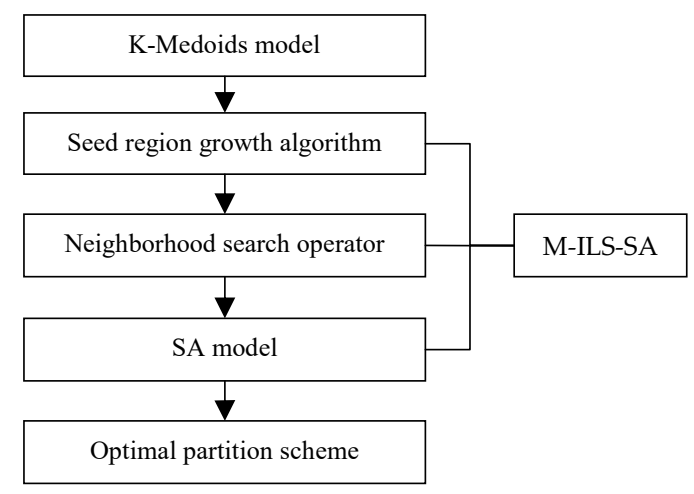

Figure 1. The basic flowchart of school district division

\section{EXPERIMENT AND RESULT ANALYSIS}

\subsection{Study Area}

In this paper, we select Liunan District of Liuzhou City, China, as a study area and conduct multi-school district division and single-school district division for public primary schools in the study area. The study area includes 447 administrative villages or community spatial units, where 42 units have primary schools. The total number of student enrollment is 44,690 . The total number of school enrollment is 47,895 . Based on the school size and class size, 18 schools are set as high-quality schools and their total enrollment is 30,090. The study area and the distribution of the schools are shown in Figure 2a. According to the actual conditions, the map of original school district division is shown in Figure 2b.

\subsection{Multi-School District Division Experiment}

Multi-school district division in the study area simulates four to eight partitions. In school grouping, for keeping the number of school enrollment in each group within a certain scale, the number of enrollment is between $60 \%$ and $140 \%$ of the average number of school enrollment and there is at least one highquality school in each group. The parameters in the proposed M-ILS-SA algorithm for multi-school district division in this experiment are set as follows: multi-start number $M=10$, neighborhood search iteration number $N=10$, SA global optimization iteration $I=10, \alpha=5$ in the objective function. The results of school grouping, initial solution, the partitions improved by neighborhood search operators, and optimized partitions by the SA algorithm are shown in Figure 3. In detail, Figure 3a shows the results of school grouping: the school units with the same color are in the same group. Figure $3 \mathrm{~b}$ shows the results of the initial partition scheme constructed by the results of school grouping, where one color is one partition. Figure $3 \mathrm{c}$ shows the results of the neighborhood search process, where the improved areas of the scheme are marked by four red rectangles. Figure $3 \mathrm{~d}$ shows the optimal partition scheme from the 10 different partition schemes after running the SA model, which is the same as the one shown in Figure 3c because it is the optimal partition scheme.
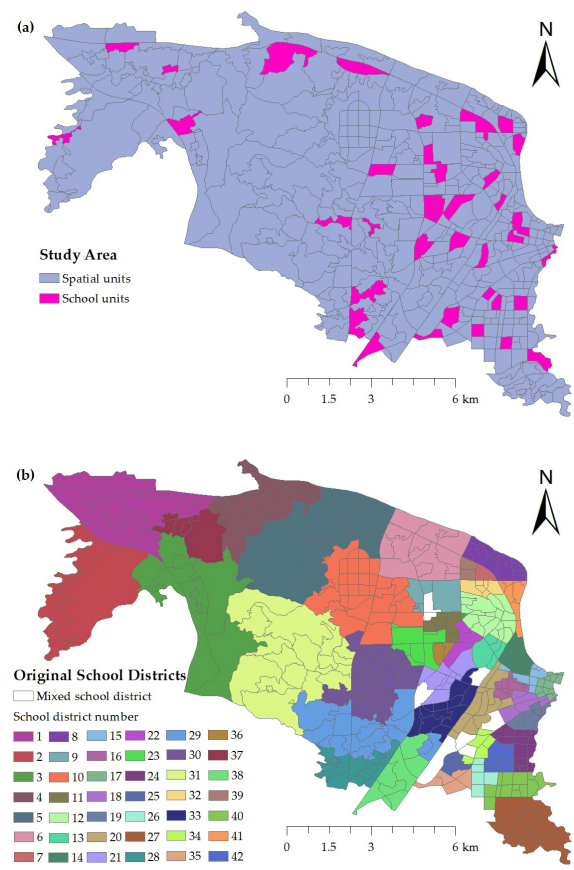

Figure 2. Study area map (a) and original school district map (b)

\subsection{Single-School District Division Experiment}

Single-school district division is a special case of multi-school district division. The number of school districts for singleschool district division is 42 because the entire study area contains 42 schools, and then each school is a group in the school grouping. In this experiment, the parameters in the proposed M-ILS-SA algorithm for single-school district division are set as follows: multi-start number $M=10$, neighborhood search iteration number $N=160$, SA global optimization iteration $I=80, \alpha=5$ in the objective function. The number of school districts in single-school district division is relatively large because if the number of iterations is too small, the neighborhood search process and the SA optimization process will be limited, and the search will not find a better solution. Therefore, in this experiment, the number of neighborhood search iterations and the SA global optimization iterations are set larger than in the above multi-school district division experiment. 

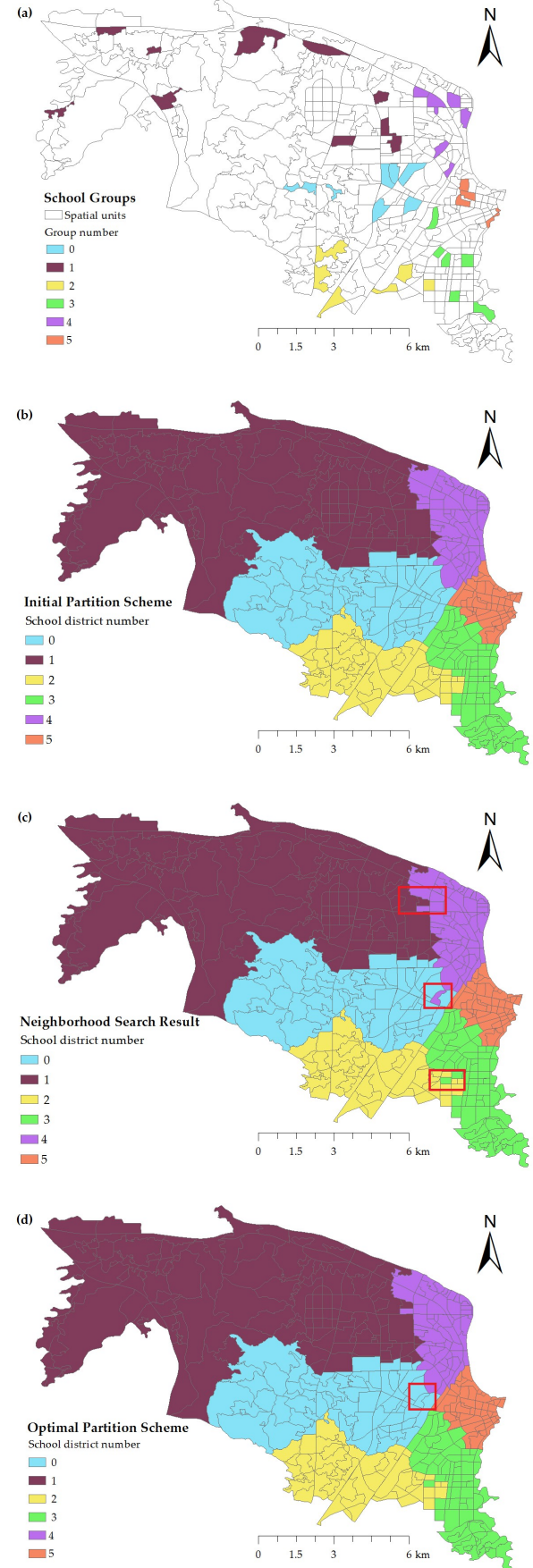

Figure 3. The results of multi-school district division: School grouping map (a), initial partition scheme map (b), map of the partitions improved by neighborhood search operators (c) and final optimal partition scheme map (d)

In the neighborhood search, if the four neighborhood search operators adopted in the multi-school district division are still to be used, then it is difficult to obtain a better solution under the current number of iterations. Moreover, if the number of search iterations is increased, the search time will become too long. Therefore, in this single-school district division experiment, we design two search operators, $(1-0)^{4}$ move and $(1-0)^{8}$ move, used for the improvement of four partitions and eight partitions, which can speed up the search and save time. Take the partition results obtained in the first run as an example. The results of school grouping, initial solution, the partitions improved by neighborhood search operators, and optimized partitions by the SA algorithm are shown in Figure 4. In detail, Figure 4a shows the results of school grouping. Figure $4 \mathrm{~b}$ shows the results of the initial partition scheme constructed by the results of school grouping, where one color is one partition. Figure $4 \mathrm{c}$ shows the results of the neighborhood search process, where the improved areas of the scheme are marked by several red rectangles. Figure $4 \mathrm{~d}$ shows the obtained partition scheme after running the SA model, where the optimized areas of the scheme are marked by three red rectangles. Since the neighborhood search process does not obtain a partition scheme that satisfies the constraints, a further optimization process by the SA algorithm is performed
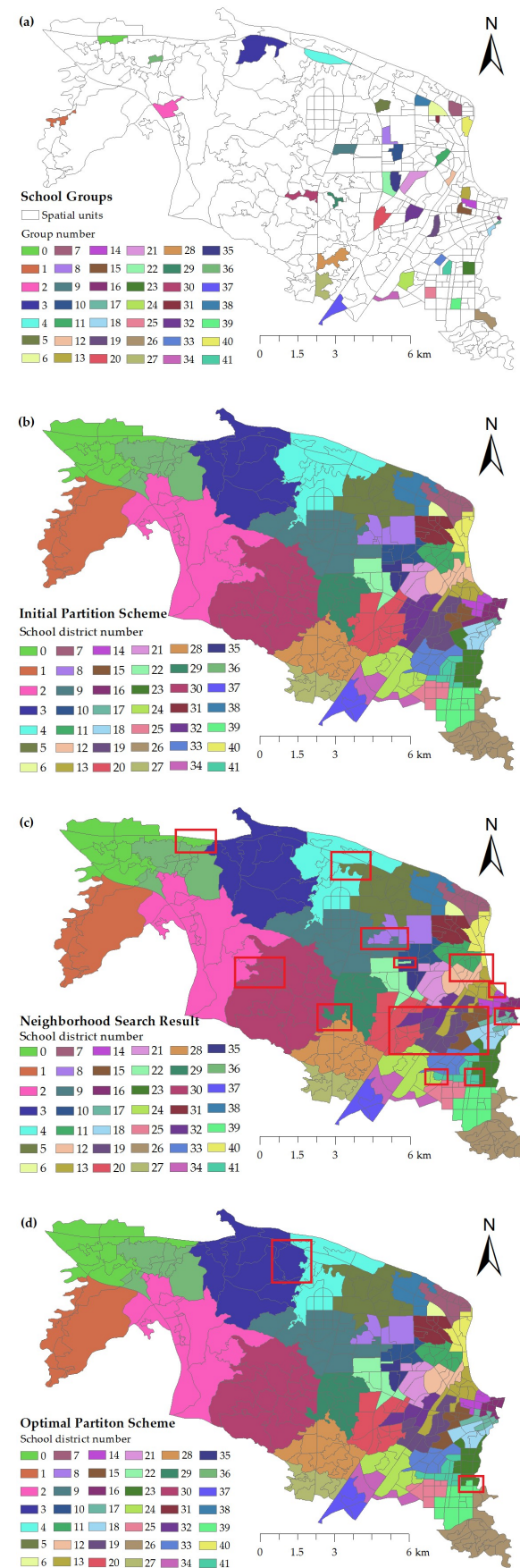

Figure 4. The results of single-school district division: School grouping map (a), initial partition scheme map (b), map of the partitions improved by neighborhood search operators (c) and final optimal partition scheme map (d) 


\subsection{Comparison and Result Analysis}

In order to improve the optimal performance for the partitioning, we used four random search mechanisms in the proposed MILS-SA algorithm. The first is random selection of the units satisfying the conditions to join the objective partition to achieve regional growth in the initial solution. The second is random selection of a region to be improved in the neighborhood search. The third is random construction of neighborhood solution of neighborhood search operators. The fourth is random selection of a high temperature region for optimization in the SA model. For the partition schemes of the study area, the proposed M-ILS-SA algorithm is run 10 times to construct different initial solutions, and then the partition optimization is carried out. The diversity mechanism can effectively avoid falling into the local optimum problem.

Figures $3 \mathrm{c}$ and $4 \mathrm{c}$ show that the neighborhood search process does not improve the individual partitions, but optimizes the partitions at a global level. This avoids the case where no partitions will be optimized after individual partitions are locally optimized. This is due to the diversity of initial solutions and the random search process that we use to achieve various results - the optimization process not only considers whether the partition to be improved is improved, but also considers whether the constructed neighborhood solution satisfies the constraints of the total student enrollment distance and the number of over-enrolled that can enable Equation (8) to evaluate the optimization process from a global perspective. Moreover, Figures 3 and 4 show that the partitioning schemes obtained by the proposed M-ILS-SA algorithm maintain spatial continuity.

A comparison between the proposed M-ILS-SA algorithm and the existing M-ILS-SPP algorithm is made under the same conditions. The comparison results of multi-school district division and single-school district division are shown in Tables 1 and 2, respectively, where DQ is district quantity, O-E is the total number of over-enrolled students in each partition, $\mathrm{D}$ is the total student enrollment distance of each unit in a partition to the nearest school, and $\mathrm{T}$ is the sum of the duration of the initial solution process, the neighborhood search process, and the SA model running process.

\begin{tabular}{|c|c|c|c|c|c|c|}
\hline DQ & \multicolumn{3}{|c|}{ M-ILS-SPP } & \multicolumn{3}{c|}{ M-ILS-SA } \\
\hline & O-E & $\mathrm{D}(\mathrm{km})$ & $\mathrm{T}(\mathrm{s})$ & $\mathrm{O}-\mathrm{E}$ & $\mathrm{D}(\mathrm{km})$ & $\mathrm{T}(\mathrm{s})$ \\
4 & 0 & 111109.05 & 504.1 & 0 & 111122.50 & 26.1 \\
5 & 0 & 93917.14 & 500.2 & 0 & 93872.95 & 28.8 \\
6 & 0 & 87251.61 & 472.1 & 0 & 87880.64 & 49.4 \\
7 & 0 & 87595.70 & 604.6 & 0 & 86680.10 & 40.2 \\
8 & 0 & 79129.41 & 576.6 & 0 & 80094.40 & 48.2 \\
\hline
\end{tabular}

Table 1. Comparison results of multi-school district division between M-ILS-SPP and M-ILS-SA

\begin{tabular}{|c|c|c|c|c|c|c|}
\hline DQ & \multicolumn{3}{|c|}{ M-ILS-SPP } & \multicolumn{3}{c|}{ M-ILS-SA } \\
\hline & O-E & D(km) & T(s) & O-E & D(km) & T(s) \\
42 & 967 & 25064.52 & 1421.6 & 120 & 25099.85 & 320.6 \\
\hline
\end{tabular}

Table 2. Comparison results of single-school district division between M-ILS-SPP and M-ILS-SA

From Tables 1 and 2, the total student enrollment distance of the M-ILS-SPP algorithm and the proposed M-ILS-SA algorithm is not much different because the partition schemes obtained by the two algorithms have spatial continuity and follow the nearby enrollment policy. However, we can also see two differences between the two algorithms:

1. Tables 1-2 show that the total elapsed time of the proposed M-ILS-SA algorithm is shorter than that obtained by the M-ILS-SPP algorithm. In the construction of an initial solution, the M-ILS-SPP algorithm only adds one unit that satisfies the conditions at each step, and thus it takes a long time. By contrast, in the proposed M-ILS-SA algorithm, we attempt to add all the adjacent unpartitioned units that satisfy the conditions into their corresponding objective partitions, and thus it accelerates the growth rate and saves a lot of time.

2. Table 2 shows that the number of over-enrolled students of the proposed M-ILS-SA algorithm is obviously less than that obtained by the M-ILS-SPP algorithm in single-school district division.

For single-school district division, we add the number of overenrolled students and the total student enrollment distance in the original school districts to show their comparisons with the ones of the M-ILS-SPP algorithm and the proposed M-ILS-SA algorithm, as shown in Figure 5, where the blue bar, red bar, and green bar represent the M-ILS-SPP algorithm, the proposed M-ILS-SA algorithm, and the original school districts, respectively. The number of over-enrolled students and the total student enrollment distance in the original school districts are statistics from the map of the original school district division shown in Figure $2 b$. The results of the proposed M-ILS-SA algorithm are less than the ones in the original school districts, and the proposed M-ILS-SA algorithm has good convergence.
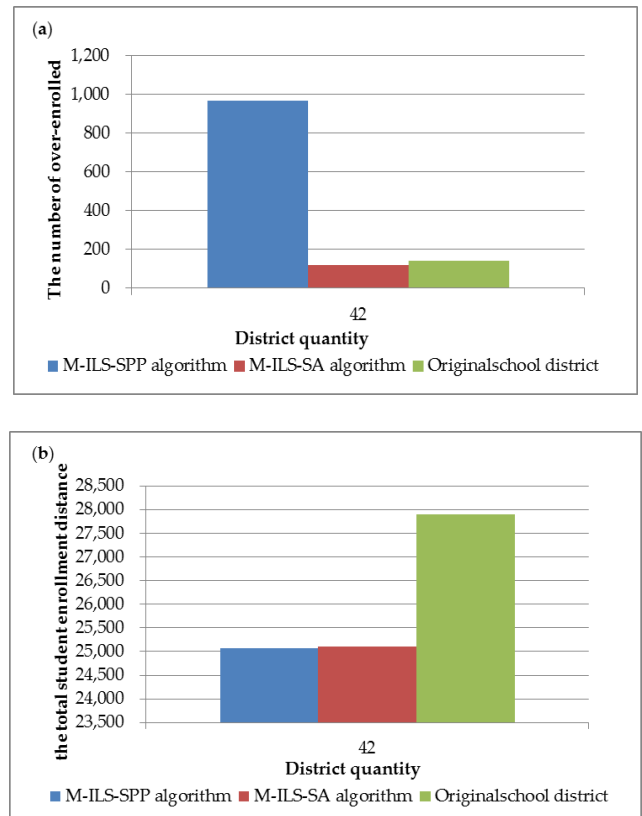

Figure 5. Comparisons of the number of over-enrolled students

(a) and the total student enrollment distance (b)

Compared with the M-ILS-SPP algorithm, the proposed M-ILSSA algorithm can greatly reduce the number of over-enrolled students in single-school district division, as shown in Figure 5a. The proposed M-ILS-SA algorithm uses the neighborhood search operators to perform a local search for partition improvement, and then combines the SA algorithm to perform global optimization again. The combination of the two 
optimization algorithms enables the proposed M-ILS-SA algorithm to find the optimal solution to a greater extent. However, the M-ILS-SPP algorithm only uses the neighborhood search operators for partition improvement in the optimization process, and thus the two algorithms exhibit difference when the number of school districts is 42 .

\section{CONCLUSIONS}

In this paper, an M-ILS-SA algorithm is proposed to solve the school district division problem. The proposed M-ILS-SA algorithm works in the framework of the M-ILS algorithm, and includes the construction of the initial solution, the design of neighborhood search operators, and global optimization and solution using the SA algorithm. Four random search mechanisms are used to achieve the diversity of the proposed M-ILS-SA algorithm, and the SA algorithm is introduced into the proposed M-ILS-SA algorithm for carrying out global optimization and solving the optimal partition scheme. An experiment shows that the proposed M-ILS-SA algorithm is suitable for both single-school and multi-school district division and has good searching ability and convergence. The following conclusions are reached:

1. In the construction of the initial solution, adding all the adjacent unpartitioned units satisfying the conditions into their corresponding objective partitions can improve the efficiency of the initial solution construction and save time.

2. In the neighborhood search process of single-school district division, the two improved neighborhood search operators $(1-0)^{4}$ and $(1-0)^{8}$ are effective at speeding up the search process.

3. The SA algorithm is introduced to improve the partition schemes from a global perspective and can effectively avoid the local optimum limitation of the local search algorithm.

4. Multiple partition schemes can be provided by using four random search mechanisms to achieve the diversity of the proposed M-ILS-SA algorithm.

5. All the partition schemes obtained by the proposed M-ILS-SA algorithm satisfy the constraints of spatial continuity.

Indeed, some practical issues, such as transportation, should be considered in the school district division problem. In this paper, we use spatial linear distance rather than road network distance as spatial distance. Therefore, future research will consider these practical issues to deal with the school district division problem.

\section{ACKNOWLEDGEMENTS}

This work was supported by the National Natural Science Foundation of China under grant number 41461085; the Natural Science Foundation of Guangxi Province under grant number 2016GXNSFAA380035; the Foundation of Guangxi Key Laboratory of Spatial Information and Geomatics under grant number 16-380-25-04; the "BaGui Scholars" Special Funds of Guangxi Province under grant number 2019-79; and the Doctoral Foundation of Guilin University of Technology under grant number 1996015.

\section{REFERENCES}

Assad, A., Deep, K., 2018. A hybrid harmony search and simulated annealing algorithm for continuous optimization. Information Sciences, 450, 246-266.

Franklin, A. D., Koenigsberg, E., 1973. Computed school assignments in a large district. Operations Research, 21(2), 413-426.

Guo, D., 2008. Regionalization with dynamically constrained agglomerative clustering and partitioning (REDCAP). International Journal of Geographical Information Science, 22(7), 801-823.

Guo, D., Jin, H., 2011. iRedistrict: Geovisual analytics for redistricting optimization. Journal of Visual Languages and Computing, 22(4), 279-289.

Jennergren, L. P., Obel, B., 1980. A study in the use of linear programming for school planning in Odense. Journal of the Operational Research Society, 31(9), 791-799.

Kim, K., Dean, D. J., Kim, H., Chun, Y., 2016. Spatial optimization for regionalization problems with spatial interaction: a heuristic approach. International Journal of Geographical Information Science, 30(3), 451-473.

Kirkpatrick, S., Gelatt, C. D., Vecchi, M. P., 1983. Optimization by simulated annealing. Science, 220, 671-680.

Ko, J., Nazarian, E., Nam, Y., Guo, Y., 2015. Integrated redistricting, location-allocation and service sharing with intradistrict service transfer to reduce demand overload and its disparity. Computers, Environment and Urban Systems, 54, 132-143.

Koenigsberg, E., 1968. Mathematical analysis applied to school attendance areas. Socio-economic Planning Sciences, 1(4), 465475 .

Kong, Y., Zhu, Y., Wang, Y., 2017. A hybrid metaheuristic algorithm for the school districting problem. Acta Geographica Sinica, 72(2), 256-268.

Lai, P. S., Fu, H. C., 2011. Variance enhanced k-medoid clustering. Expert Systems With Applications, 38(1), 764-775.

Liu, S., Wu, W. H., Kang, C. C., Lin, W. C., Cheng, Z., 2015. A single-machine two-agent scheduling problem by a branchand-bound and three simulated annealing algorithms. Discrete Dynamics in Nature and Society, 2015, 1-8.

Mckeown, P., Workman, B., 1976. A study in using linear programming to assign students to schools. Interfaces, 6(4), 96101.

Openshaw, S., 1977. A geographical solution to scale and aggregation problems in region-building, partitioning and spatial modelling. Transactions of the Institute of British Geographers, 2(4), 459-472.

Ricca, F., Simeonea, B., 2008. Local search algorithms for political districting. European Journal of Operational Research, 189(3), 1409-1426 
Rincón-García, E. A., Gutiérrez-Andrade, M. A., de-los-CobosSilva, S. G., Lara-Velázquez, P., Ponsich, A. S., Mora-Gutiérrez, R. A., 2013. A multiobjective algorithm for redistricting. Journal of Applied Research and Technology, 11(3), 324-330.

Rincón-García, E. A., Gutiérrez-Andrade, M. A., de-los-CobosSilva, S. G., Mora-Gutiérrez, R. A., Ponsich, A. S., LaraVelázquez, P., 2017. A comparative study of population-based algorithms for a political districting problem. Kybernetes, 46(1), 172-190.

Schoepflea, O. B., Churchb, R. L., 1991. A new network representation of a "classic" school districting problem. Socioeconomic Planning Sciences, 25(3), 189-197.

Sheng, W., Liu, X., 2006. A genetic k-medoids clustering algorithm. Journal of Heuristics, 12(6), 447-466.

Taylor, R. G., Vasu, M. L., Causby, J. F., 1999. Integrated planning for school and community: The case of Johnston County, North Carolina. Interfaces, 29(1), 67-89. 